\title{
Survey on the Role of IoT in Intelligent Transportation System
}

\author{
H. Varun Chand, J. Karthikeyan \\ VIT University, Vellore, Tamil Nadu, India
}

\begin{tabular}{|c|c|}
\hline Article Info & ABSTRACT \\
\hline Article history: & Precise and appropriate traffic related data allows travellers to choose \\
\hline Received Dec 8, 2017 & $\begin{array}{l}\text { suitable travelling modes, travelling paths, and departure time, which is } \\
\text { crucial for the success of Intelligent Transportation System (ITS). With the }\end{array}$ \\
\hline Revised Jan 9, 2018 & growth of vehicles, the rate of pollution and consumption of fuel has \\
\hline Accepted Jan 14, 2018 & $\begin{array}{l}\text { increased, it also creates traffic congestions. For the recent years there has } \\
\text { been a rapid growth in technology, which can be explored to solve traffic }\end{array}$ \\
\hline Keywords: & $\begin{array}{l}\text { issues. However, depending upon the available technologies each } \\
\text { countries ITS research area may be different. The objective of this literature }\end{array}$ \\
\hline Intelligent Trans portation & review is to integrate ITS with internet of things and it also discusses \\
\hline System, & $\begin{array}{l}\text { the prospect of clustering, controller system, location identification and } \\
\text { resource privacy in ITS. }\end{array}$ \\
\hline $\begin{array}{l}\text { Internet Of Things, Clustering, } \\
\text { Resource Privacy. }\end{array}$ & \\
\hline Vanet, & $\begin{array}{r}\text { Copyright (c) } 2018 \text { Institute of Advanced Engineering and Science. } \\
\text { All rights reserved. }\end{array}$ \\
\hline \multicolumn{2}{|l|}{ Corresponding Author: } \\
\hline $\begin{array}{l}\text { H. Varun Chand, } \\
\text { VIT University, } \\
\text { Vellore, Tamil Nadu, India. } \\
\text { E-mail:varunchand.h2016@vits }\end{array}$ & ac.in \\
\hline
\end{tabular}

\section{INTRODUCTION}

An intelligent transportation system (ITS) is an application that provide inventive utility relating to different ways of transport and traffic management, which allows various users to make safer, more synchronized, and smarter use of transportation networks. This paper focuses on to the importance of IoT in intelligent transportation systems. It can be achieved with the help ongoing informations generated from sensors, auto control networks and computer processing systems. ITS with the integration of Internet of Things (IoT) has got its initial stages in many applications, and this will provide significant transformation in next few years [1].

For the past serveral years the growth rate of vehicles has increased rapidly, which results in serveral problems like traffic congestions, increased rate of carbon emission, increased rate of fuel consumption etc. This paper addresses the role of IoT in managing various problems in Inteligent transportation systems.

\subsection{Integrating Intelligent Transportation System with Internet of Things (IoT)}

The traffic system will be benefited if ITS can be integrated with Internet of Things. In the future applications, IoT will play crucial role in the case of normal operation of ITS [2]. The various applications of IoT in ITS includes, theft vehicle detecting system, prioritized vehicle scheduling, non-stop toll collecting system, traffic violation monitoring, etc. Traffic flow prediction based on historical data, various Multivariable Phase Space Reconstruction methods etc helps in reducing traffic congestions [3]. In the future, we can envision the connection between automobiles and people, then between roads and vehicles as a big system to resolve a lot of traffic congestions, air pollution and providing safe and secure transportation.

\subsection{Traffic optimization in ITS}

A huge amount of traffic related data are generated and collected from various sensors, radars, cameras, mobile GPS, inductive loops etc. We have entered into a period of transportation in big data with 
the emergence of various sensor units and other technologies. In the earlier days, shallow traffic models were used for traffic managment but are not pleased. In recent years, machine learning based deep learning approaches are used and it has been functional through the achievement in natural language processing, dimensionality reduction, classification tasks, object detection, and motion modelling. The deep learning algorithms exploit various layer constructions or deep designs to remove intrinsic topographies in files in the minimum range to the maximum range, and it may be determined by the maximum quantities of arrangement in the records [4].

\section{REVIEW PLAN}

In this paper, we discussed the different approaches and techniques in the area of traffic optimization by integrating it with Internet of Things (IOT). Tarffic optimization helps to reduce the time wasted on road traffic, reduces air pollution rate, reduces fuel consumption etc. So people are very much troubled with traffic congentions, sharing their sensitive information etc. This literature surevy paper focuses on the existing literature presented in the field of Intelligent Transportation System.

\subsection{Clustering and Control System}

Clustering process starts by selecting a cluster head $(\mathrm{CH})$, which sends clustering information from one vehicle to another vehicle. By this way, it provides lesser cost and ease transportations. The $\mathrm{CH}$ combinations act as a communication point among different vehicles and amoung memberships of other groups. This $\mathrm{CH}$ can find the position of its memberships and create an overview of its group condition. A system controller modules and hardware components are been used by the control systems unit. The hardware component helps to optimize the traffic congentions. It describes numerous connection models to optimize the unpredicted traffic issues (Figure 1).

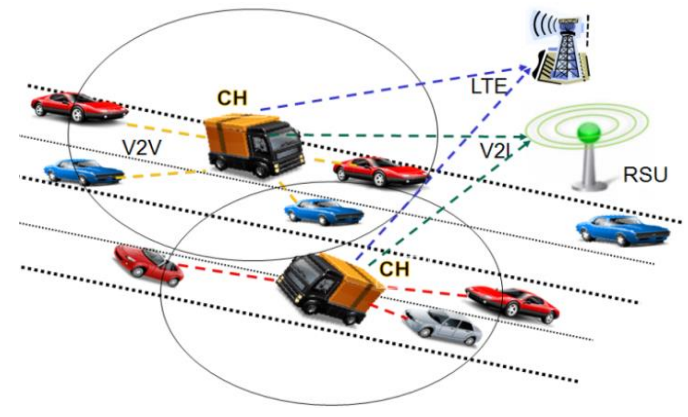

Figure 1. Clustering strategy in ITS

Context: In the development of ITS, VANETs provide important benefits on the dynamic and portioned network topology, and this topology gives the significance of rapid movement of vehicles. Now, clustering is extensively castoff as the regulator arrangements to create VANET topology fewer active for security, MAC and routing protocols [5].

Objective: To generate vehicular networks, Adaptive Weighted Clustering Protocol (AWCP) were depicted, and these techniques may take the route of automobiles, its fastness and the amount of neighboring automobiles, highway ID and position. This increased the network stability.

Methods: Though, the numerous regulator constraints of our AWCP, limitation tuning becomes the main problem in our method. In the case of optimization procedures, they defined a multi-objective task whose contributions are the AWCP's limits, and their goals includes: maximizing data delivery rate, providing stable cluster structures and dropping the grouping overhead. They addressed this problem through the Non-dominated Sorted Genetic Algorithm version 2 (NSGA-II). They assessed and matched their presentation through additional multi-objective optimization procedures as Multi-objective Differential Expansion (MODE) and Multi-objective Particle Swarm Optimization (MOPSO).

Results: The tests described that the NSGA-II increases the consequences of MODE and MOPSO regarding the ratio of non-dominated results, spacing, and opposite relating to distance.

Significance: VANETs channel efficiency could be enhanced through the growth of cross-layer architecture were all $\mathrm{CH}$ are accountable for the transmission of bandwidth to each of its group members.

Drawback: These procedures do not generate a constant architecture for clustering. 
Context: The manufactural and theoretical requirement was to arrive big data era in various computer software fields and embedded system. The significant trial is to attain the transportation movement radiances skilfully via the collected energetic Automobile Association data scale [6].

Objective: The Objective was to make a ITS reliable by using a NeverStop strategy which uses fuzzy control methods and genetic algorithms. To control the traffic lights, NeverStop has constructed in automatically manner.

Methods: Here to adjust the traffic lights waiting time, they used a genetic algorithm and fuzzy control method. Throughout this technique, the running time was significantly reduced with the EBox-II terminal device. In the case of controller module, initially used the phase model and then used the multiple intersection models and single intersection model. Lastly, distinct approaches were presented towards usage of unpredicted circumstances.

Results and significance: Outcomes in the model validate that NeverStop could significantly assist researchers towards decreasing the normal waiting time of automobiles.

Drawback: This concept lonely deliberates the nearperfect readings of the sensor, and this is not reliable for the recent trends.

Contex: Emergency vehicles are transported with high priority, without allowing them to wait in traffic congetions. This can be done with the help of RFID tags attached to these vehicles [7].

Objective: To make smooth transportation on the emergency vehicles and managing intelligent traffic control system.

Methods: All separate automobile was furnished through superior (RFID) radio frequency identification tag, it helps them to track their presence at various traffic check points. They use NSK EDK125-TTL, RFID reader, and PIC16F877A chip for reading the RFID labels devoted to the system. It deliberates the amount of cars that permits on a specific trail throughout a quantified period. It also regulates the system jamming. When the RFID-tag-read goes to the pinched automobile, formerly information was directed by GSM SIM300 to the control room.

Results: This unit uses ZigBee components on PIC16F877A and CC2500 system-on-chip for communication among the traffic manager. The technique was verified in altered groupings of the system workshop.

Significance: The particular position of given automobile is recognized. Presently, they had realized arrangement by sighted one street of the circulation connection.

Drawback: It could be industrialized through spreading to altogether the infrastructures in a multiroad connection.

\subsection{Resource privacy:}

To adopt the new technique, user's privacy violations were introduced with some security concerns. To emerge security in ITS, some privacy-preserving mechanisms were used for the reliable transportation in ITS (Figure 2).

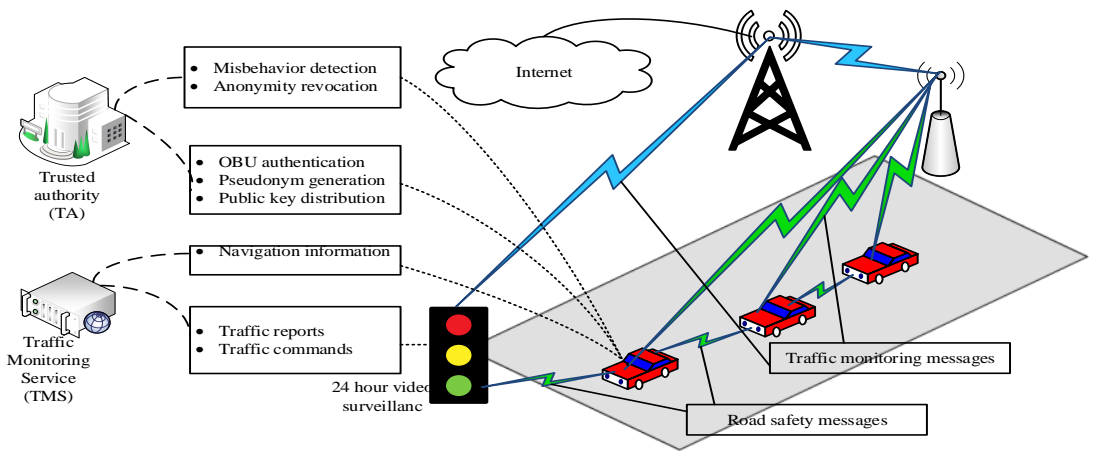

Figure 2. Schematic representation of resource privacy in ITS

Context: Privacy protection is more important than anything else for the emergence of Intelligent Transportation System (ITS) applications, such as transportation managing and highway security. These applications need systematic communication of messages between various automobiles or between automobiles and back-end servers. The received messages should be verified so that messages from harmful or crashing entities can be recognized and that must be removed [8]. 
Objective: To provide privacy among automobiles and a trusted authority (TA), using cryptographic algorithms.

Methods: This work was based on the independent privacy-protecting authentication arrangement where automobiles alone requires to interact the TA; later, they could reintroduce the pseudonyms int eractive using TA. To the best of our information, this is the first verification arrangement given that automobiles with the proficiency to reintroduce their pseudonym groups deprived of demanding perpetual interaction with a TA.

Significance: Some of the advantages were: 1) it permits an independent working method for belongings while the TA is not obtainable over BS or RSU, and 2) diminishes its transmission overhead meanwhile the automobiles were not essential to create a point-to-point protected connection through TA.

Drawback: The issuers were not providing any anonymity revocation arrangement, and henceforth this scheme is unable to deliver non-rejection of communications.

Context: In transportation systems, Cyber-security affects the gain access and variety of interfaces. Such pressures become broader more challenging with the appearance of automobile-to-infrastructure (A2I) and automobile-to-automobile (A2A) communication technologies [9].

Objective: To provide the safety and security transportation models, this work used formal verification, hybrid modelling and automated synthesis techniques.

Methods: They used mutual adaptive cruise control towards demonstrating the efficiency of the joint synthesis and verification framework for investigating the conveyance systems. Here, the computation, embedded control and transmission mechanisms are carefully interrelated through the substantial, mechanical and environment mechanisms at present. Consequently, it was significant towards the addressed abovementioned safety concerns, then similarly significant safeguard the practical safety events. For example, scheduling restraints are not measured through the request of safety processes.

Significance: This work allows these joined repetitions among synthesis and verification through handling and explaining the interchange of timing restraints over a recognized interface.

Drawback: The plan is not manufactured based on the assumption of timing restrictions, then the unstable constraint was approved back to the confirmation layer.

\subsection{Location Identification:}

Location identifiction in ITS depends on the Received Signal Strength Indication (RSSI). Based on this RSSI, they can track and detect the location of the vehicles.

Context: A Recent study in ITS inflections on Automatic Incident Detection (AID) methods is provided. By an advance in sensor and networking, current automobiles have the skill to broadcast through further RSUs for improving road safety [10].

Objective: These novelties in transport skill deliver transportation managing scheme uses traffic data, which is collected from various automobiles on the highways to identify congestion and rate of traffic.

Methods: V2I interface was used for information gathering, and they studied the usage of new limits in combination through AID. Precisely, they deliberate the detachment automobiles requirement to an alternate path and at the alteration in automobiles velocity while altering paths in event and non-event circumstances, it used for establishing procedures for AID. The usage of such new parameters in AID is novel and allows as sociation of detachment and velocity through sense events.

Results: Alternative AID methods over advanced event recognition proportions, around 25\% smaller peak line standards and $20 \%$ earlier degeneracy of highway congestion.

Significance: It assumes that a V2I model was castoff for statistics group, and then they studied the routine conjunction with AID.

Drawback: Approximation of the on position centred on the composed lane varying data is not conceivable here.

Context: Dynamic congestion pricing and traffic routing systems were receiving a position in recent research. Path forecast and vehicle intensity approximation was a significant constituent of such schemes [11].

Objective: To predict a number of paths on the highway, and automobile's location in those paths through video recorder taken through a dashboard camera.

Methods: Here they use closed-loop strategy and occurrence of automobiles to support the assignment of self-positioning. The combination of numerous issues and high-level semantic information on the solution can be expressed as a difficult situation with the help of a Bayesian outline. Here, a number of paths, the vehicle's location in those paths and the occurrence of additional automobiles are measured as parameters. In addition to this, bounding box selection arrangement decreases the number of incorrect recognitions and increase the computational competence. 
Results: The consequence demonstrates that there is a huge decline in the number of incorrect recognitions. The whole method was verified on real-world tapes and was originating towards given satisfactory consequences.

Significance: Huge fall in travel delays and congestion cost. They also progressed a selection on bounding box criteria, which could be functional to a comprehensive set of containers or the containers received from additional box-proposal approaches.

Drawback: The scheme currently practices unbiased video data to create calculations.

Context: Dissimilar wireless transmission skills, such as $\mathrm{Wi}-\mathrm{Fi}$, worldwide interoperability and $4 \mathrm{G}$ cellular technologies for WiMAX had been used as replacements or enhancement to wired interaction in ITS [12].

Objective: To attain an anticipated range of presentation necessities among neighbouring nodes, smallest system presentation inceptions must be comprehended in the field.

Methods: Consequently, the incentive of this work was to progress approaches to estimate transmission presentation of wireless access points (APs) for the original application which is possible and dependable in secondary ITS applications. Field trials exposed that transmission power, modulation rates, the distance between nodes and line of sight plays a cruisal job in the presentation of wireless transmission in dissimilar roadway circumstances.

Results: Transport activities could classify the attainable presentation, such as delivery ratio, flooded throughput and the received signal strength at a specific site, through succeeding the arena test process industrialized in this research.

Significance: The as sessment plans and consequences obtainable in this work were contributed for the future planning and designing of wireless transmission for a roadway wireless sensor networks (WSN).

Drawback: The great potential in $\mathrm{WiFi}$ data transmission was not appropriate for small inactivity applications.

\section{PROSPECT OF ITS IN IOT}

The expansion of IoT is a continous procedure and a lot of difficulties are still to be solved. The is sues includes less power nodes and computing, self-organized scattered schemes knowledge, least cost and least latency interaction, credentials and setting tools, and scattered communication [13].

The IoT generates a prospect for the internet-based facilities, there by augmenting the profitable and communal possible future of IoT. The growth of IoT saves successfully onward along the scale, combined, and smart. Encouraged through knowledge, calibration, and request experiences, IoT requests would enlarge the measure in the dissimilar productions, and additional originalities would be involved to come in. By the growth of intelligent data holding ability, IoT organizations will identify ample access over data sharing and its association. The growth of an ideal scheme would inevitably take internet of things (IoT) focused on regular life. The IoT produces a prospect for internet-based provision, expanding the success and communal possible destiny of IoT.

Information interoperability would yield place amongst dissimilar belongings, diverse initiatives, altered businesses, and dissimilar areas. The application models will adjust from closed to open and the globalisation of IoT application scheme allocation for dissimilar trades and fields can be built. A systematic method is needed for tackling and resolving the interoperability of IoT plans and facilities at countless deposits.

The IoT would take unified commercial and communal interacting over fast dependable and protected systems into our society. The environment consciousness and inter-things data conversation are the key ideas for the expansion of IoT. Collecting and regulating the intelligence at the scheme level would be important for the investigation, i.e., the fusion of sensors and multi-standard actuators, adaptive communication subsystems, adaptable antennae etc.

In the upcoming years, energy-saving and self-sustainable systems will play pivotal role in increasing issues to the IoT. The method of gathering energy from environments must be expanded. Efficiency in management and communication must be improved through new circuits, new programming pattern, and the further development of energy-saving protocols and smart antennae. Charging of global IoT terminals, the power utillization of international IoT access points and gateways, will be one of the dominant power buyers in the future world.

\section{CONCLUSION AND FUTURE WORK:}

The traffic problems may differ for different countries according to the case of fund investment and present technology used. So the development level of ITS and its research fields are distinct. This paper 
comsided the traffic problems as not only problems of individual countries, but also a global problem. By integrating IoT with ITS we can improve the performance of traffic optimization. The IoT device collects, stores and process faster way of traffic informations, which help in optimizing the traffic problems. Future services may include location-sensitive billing, location-based advertising, and information services such as navigation, points of interest etc. These systems provide more services and are less complex. We hope that the use of various IoT devices and differnt techniques will make our transportation systems more efficient and secure, with less congestion, pollution and optimal use of fuel.

\section{REFERENCES}

[1] Ning, Huansheng, Hong Liu, Jianhua Ma, Laurence T. Yang, and Runhe Huang. Cybermatics: Cyber-physicalsocial-thinking hyperspace based science and technology. Future Generation Computer Systems, Vol. 56, 2016, pp. 504-522.

[2] Juan Antonio Guerrero-ibanez, Sherali Zeadally and Juan Contreras Castillo. Integration challenges of intelligent transportation systems with connected vehicle, cloud computing, and internet of things technologies. IEEE Wireless Communications, Vol. 22, No. 6, 2015, pp.122-128.

[3] Duo Zhang and Fengqing Han. Short-term Traffic Flow Prediction Based on Multivariable Phase Space Reconstruction and LSSVM, Indonesian Journal of Electrical Engineering, Vol. 12, No. 9, September 2014, pp. 6946-6953

[4] Lv, Yisheng, Yanjie Duan, Wenwen Kang, Zhengxi Li, and Fei-Yue Wang. "Traffic flow prediction with big data: a deep learning approach." IEEE Transactions on Intelligent Transportation Systems 16, no. 2 (2015): 865-873.

[5] Hadded, Mohamed, Rachid Zagrouba, Anis Laouiti, Paul Muhlethaler, and Leila Azouz Saidane. A multi-objective genetic algorithm-based adaptive weighted clustering protocol in vanet. IEEE Congress on Evolutionary Computation (CEC), 2015, pp. 994-1002.

[6] Wang, Chao, Xi Li, Xuehai Zhou, Aili Wang, and Nadia Nedjah. Soft computing in big data intelligent transportation systems. Applied Soft Computing, Vol. 38, 2016, pp. 1099-1108.

[7] Sundar, Rajeshwari, Santhoshs Hebbar, and Varaprasad Golla. Implementing intelligent traffic control system for congestion control, ambulance clearance, and stolen vehicle detection. IEEE Sensors Journal, Vol. 15, No. 2, 2015, pp. 1109-1113.

[8] Wang, Changii, Dongyuan Shi, Xilei Xu, and Jian Fang. An anonymous data access scheme for VANET using pseudonym-based cryptography. Journal of Ambient Intelligence and Humanized Computing, Vol. 7, No. 1, 2016, pp. 63-71.

[9] Zheng, Bowen, Wenchao Li, Peng Deng, Leonard Gerard, Qi Zhu, and Natarajan Shankar. Design and verification for transportation system security. In Proceedings of the 52nd Annual Design Automation Conference, ACM, 2015, pp. 96.

[10] Popescu, Otilia, Sarwar Sha-Mohammad, Hussein Abdel-Wahab, Dimitrie C. Popescu, and Samy El-Tawab. Automatic Incident Detection in Intelligent Transportation Systems Using Aggregation of Traffic Parameters Collected Through V2I Communications. IEEE Intelligent Transportation System, Vol. 9, No. 2, 2017, pp. 64-75.

[11] Chandakkar, Parag S., Yilin Wang, and Baoxin Li. Improving vision-based self-positioning in intelligent transportation systems via integrated lane and vehicle detection. Applications of Computer Vision (WACV), 2015 IEEE Winter Conference, 2015, pp. 404-411.

[12] Zhou, Yan, Kakan Chandra Dey, Mashrur Chowdhury, and Kuang-Ching Wang. Process for evaluating the data transfer performance of wireless traffic sensors for real-time intelligent transportation systems applications. IET Intelligent Transport Systems Vol. 11, No. 1, 2016, pp. 18-27.

[13] Chen, Shanzhi, HuiXu, Dake Liu, Bo Hu, and Hucheng Wang, A vision of IoT: Applications, challenges, and opportunities with china perspective, IEEE Internet of Things Journal, Vol. 1, No. 4, 2014, pp. 349-359. 